
\title{
28 Research Square \\ Initial Pulse Pressure and Shock Index Predict Mortality in Burn Patients
}

Alexandra Pires-Menard ( $\square$ alexandra.pires-menard@uhsinc.com )

Department of Internal Medicine, Temecula Valley Hospital, Temecula, CA, USA

\section{Fanglong Dong}

Department of Statistical Sciences, Western University of Heath Sciences, Pomona, CA, USA

\section{Richard Jin}

Department of Internal Medicine, Temecula Valley Hospital, Temecula, CA, USA

\section{David Lee}

Department of Critical Care Medicine, Temecula Valley Hospital, Temecula, CA, USA

\section{Stathis Poulakidas}

Department of Trauma and Burn Surgery, John H. Stroger Hospital of Cook County, Chicago IL, USA

Faran Bokhari

Department of Trauma and Burn Surgery, John H. Stroger Hospital of Cook County, Chicago IL, USA

\section{Research Article}

Keywords: Shock Index, Pulse Pressure: Burn Injuries, Burns

Posted Date: February 24th, 2022

DOI: https://doi.org/10.21203/rs.3.rs-1356323/v1

License: (c) (i) This work is licensed under a Creative Commons Attribution 4.0 International License. Read Full License 


\section{Abstract}

\section{Background:}

Burn injuries result in profound hemodynamic and cardiovascular dysfunction. Pulse pressure (PP) and shock index (SI) can be used to guide medical management in acute trauma settings however its application in acute burn settings is unknown. The purpose of this study was to determine whether pulse pressure and shock index on admission were early predictors of in-hospital mortality in burn patients.

\section{METHODS:}

This retrospective study analyzed burn patients admitted to John H. Stroger Hospital of Cook County in Chicago, Illinois, USA from 2013-2019. Inclusion criteria were patients aged $>1$ year with TBSA values $\geq$ $15 \%, 2$ nd and 3rd degree thermal injuries. Exclusion criteria included non-thermal burns, missing values and those dead on arrival.

Variables studied were age, gender, TBSA, inhalation injury, Baux score, R-Baux score and vital signs (systolic blood pressure, diastolic blood pressure and heart rate) on arrival. SI and PP values were calculated from the vital signs. Baux and R-Baux score were calculated from data in the electronic medical records.

Independent t-test compared mortality outcomes in survivors versus non-survivors. Pearson's Chi square test compared mortality outcomes associated with gender and inhalation injury status. Adjusted odds ratio with corresponding 95\% confidence intervals were calculated for R-Baux and SI.

\section{RESULTS:}

108 burn patients were included in this study. Mean age was 40 years and 68 patients (64\%) were male. Mean TBSA was $30 \%$ and inhalation injury was present in $30 \%(32 / 106)$ of the patients. Among eighteen non-survivors, fourteen (78\%) had inhalation injury. For all patients, the mean Baux and R-Baux score were 70 and 75 , respectively. Overall mortality rate was $17 \%$.

On bivariate analysis, mean $\mathrm{SI}$ in survivors and non-survivors was 0.7 and 1.0. Mean PP in survivors and non-survivors were $61 \mathrm{mmHg}$ and $47 \mathrm{mmHg}$.

Adjusted odds ratio $(95 \% \mathrm{Cl})$ for R-Baux and SI were 1.06 (1.03-1.09) and 26.48 (4.34-161.46), respectively.

\section{CONCLUSION:}

A lower PP and a higher SI on admission are predictors of mortality in patients with significant burns. SI is associated with 26-times higher likelihood of mortality. These initial parameters can help identify patients that may require close monitoring and aggressive resuscitation to improve survival rates in burn victims. 


\section{Background}

Burn injuries are a leading cause of morbidity and mortality in patients worldwide ${ }^{1}$. According to the World Health Organization, burn injuries attribute to more than 180,000 deaths annually worldwide ${ }^{1}$. Despite increased public awareness and prevention efforts, the American Burn Association (ABA) estimates that 450,000 patients per year require treatment for burn-related injuries with 30,000 requiring admission to burn centers ${ }^{2}$. Although $96.7 \%$ of patients treated in burn centers survive ${ }^{3}$, non-fatal burn injuries can result in decreased quality of life with devastating life-long sequela of emotional and physical disabilities ${ }^{1}$.

Burns are acute tissue injuries resulting from exposure to heat, electricity, chemicals, radiation or friction. The majority of injuries result from thermal burns ${ }^{4}$, however most burn fatalities result from smoke and toxic gas inhalation associated with thermal burns ${ }^{5}$. Thermal burns can cause both local injury to the skin from contact with hot surfaces, liquids, flame or steam and if severe, can also induce a systemic response ${ }^{4}$.

The local response in the skin results in three concentric zones of injury first described by Jackson in 1947 as the "zones of coagulation, stasis and hyperemia" ${ }^{6}$. The zone of coagulation is the inner-most region including the primary site of injury nearest to the heat source resulting in maximal damage and irreversible tissue necrosis ${ }^{6}$. Surrounding this area is the zone of stasis characterized by potentially viable tissue resulting from possible reversible hypoperfusion-inducing-ischemia ${ }^{6}$. The outermost zone of hyperemia is an area characterized by reversible vasodilatation resulting in increased tissue perfusion ${ }^{6}$.

Tissue injury from burn mediated damage immediately triggers the inflammatory cascade via the activation of a master transcription activator protein, nuclear factor $\mathrm{kB}$ required for wound repair and healing ${ }^{7}$. However, severe burn injury can lead to upregulation of the inflammatory cascade triggering an extensive systemic response which through various mechanisms ultimately induces an immunocompromised-like state in conjunction with a hypermetabolic state predisposing the burn victim to shock, sepsis, multiple organ failure and death ${ }^{7}$. During this initial phase of the burn injury, the interaction between pro-inflammatory and vasoactive mediators (including prostaglandins, IL- 6 and TNFa, histamine) in combination with stress-induced catecholamine release leads to increased capillary permeability, massive intravascular protein and fluid volume deficits and edema formation resulting in profound hemodynamic and cardiovascular dysfunction ${ }^{4,8,9}$ commonly known as burn shock $^{8,10}$.

Burn shock is initially associated with decreased cardiac output as a consequence of decreased preload, with increased afterload and decreased myocardial contractility ${ }^{11}$. Early and effective fluid resuscitation with close monitoring is the cornerstone of preventing and treating shock in burn victims. The Parkland formula is commonly utilized to administer adequate amounts of fluid and prevent complications associated with overzealous administration of fluids in burn victims ${ }^{11,12}$. Although it is gold-standard for approximating early fluid needs in burn victims, it does not account for changes in hemodynamic status 
that may occur during the initial burn injury stage. Fluids must be adjusted accordingly with close monitoring. With early and appropriate treatment of burn shock, the survival rate in burn patients drastically improves ${ }^{11,13}$.

Shock index (SI) was first introduced in 1967 by Allgower and Buri as a simple and effective tool to predict hypovolemic shock severity ${ }^{14}$. SI is calculated by dividing the heart rate (HR) by the systolic blood pressure (SBP), with a normal range currently accepted as $0.5-0.7$ in healthy adults with evidence suggesting that values up to 0.9 are within the upper limit of normal; however, poorer outcomes associated with SI values $\geq 1.0$ are indicative of worsening hemodynamic status and occult shock. Due to the nature of its linear inverse relationship with hemodynamic parameters including cardiac index, stroke volume, left ventricular stroke work and mean arterial pressure ${ }^{14}, \mathrm{SI}$ has been demonstrated to predict mortality and other severe outcomes including the necessity of intensive care unit admission and need for blood transfusion in trauma, postpartum hemorrhage, acute myocardial infarction, stroke and septic patients ${ }^{15}$; however, its role in predicting mortality outcomes in burn victims remains unknown.

Similarly, pulse pressure is another effective tool for approximating hemodynamic status. Pulse pressure is defined as the difference between the systolic and diastolic blood pressure, with normal values ranging from $40-60 \mathrm{mmHg}$ in healthy adults. Low or narrow pulse pressures ( $<40 \mathrm{mmHg}$ ) occur when the value is less than $25 \%$ of the systolic value and because systemic pulse pressure is proportional to the stroke volume $^{16}$, a low or narrow pulse pressure indicates insufficient preload and reduced cardiac output suggestive of cardiogenic or hypovolemic shock from significant volume loss. Multiple studies have shown that narrow pulse pressure in seemingly hemodynamically stable patients is an independent early predictor of active hemorrhage and impending shock.

Pulse pressure and shock index have been extensively studied as prognostic tools that can help identify patients at risk for impending hemodynamic collapse and guide medical management in acute trauma, hemorrhage and septic settings; however, its application in acute burn settings has yet to be explored. The purpose of this study was to determine if hemodynamic physiological changes, such as shock index and pulse pressure, in response to significant thermal burn injuries, predict in-hospital mortality.

\section{Methods}

This was a retrospective cohort study in burn patients admitted to John H. Stroger Hospital of Cook County in Chicago, Illinois, USA from 2013-2019. Inclusion criteria were patients aged $>1$ year with isolated major burn injuries corresponding with Total Body Surface Area (TBSA) values $\geq 15 \%$, 2nd and 3rd degree thermal injuries, those transferred or directly admitted to Cook County Hospital. Exclusion criteria included patients with missing values, non-thermal burns, trauma injuries, and dead on arrival.

Burn patients were dichotomized into two groups: survivors and non-survivors. Variables compared between these groups collected from patient electronic medical records included age, gender, TBSA, inhalation injury status and vital signs [systolic blood pressure (SBP), diastolic blood pressure (DBP), and 
heart rate (HR)] on arrival. Pulse pressure (PP) and shock index (SI) were calculated from the patients' recorded vital signs on arrival to Cook County Hospital. For patients that were transferred to Cook County Hospital, the documented initial vital signs from the transferred institution's admission note were utilized to determine the pulse pressure and shock index on arrival. The formula used to calculate pulse pressure on arrival was:

\section{Pulse Pressure (PP) = SBP - DBP}

Shock index on arrival was calculated with the following formula:

\section{Shock Index (SI) = HR/SBP}

In addition, the following variables Baux score and revised Baux score (R-Baux) were also calculated from patient data with the following formulas:

\section{Baux score $=$ TBSA + age}

\section{R-Baux score $=$ TBSA + age $+17 R$}

\section{$R=$ inhalation injury status $(R=1$ if inhalation injury, $R=0$ if no inhalation injury)}

We decided to utilize the R-Baux score for mortality prediction in all age groups as opposed to implementing the Pediatric-Baux score in children $<15$ years because R-Baux is well-known for its robustness with acceptable validity and accuracy in predicting burn victim mortality outcomes, unlike PBaux ${ }^{17}$.

Summary statistics were reported as mean [standard deviation (SD)]. On bivariate analysis, the independent t-test was utilized to analyze mortality outcomes associated with continuous variables including: age, TBSA, Baux score, R-Baux score, SBP, DBP, HR, pulse pressure and shock index. Pearson's Chi square test was utilized to analyze mortality outcomes associated with gender and inhalation injury status. Multivariate logistic regression analysis was used to calculate adjusted odds ratio with corresponding 95\% confidence intervals for both variables R-Baux score and shock index. Statistical analysis was performed using STATA for Windows and R version 4.0.5, 2021 (R Foundation for Statistical Computing) 2021 for MacOS. Cook County Hospital IRB approval for study 19-154 was obtained.

\section{Results}

108 burn patients were included in this retrospective cohort study at Cook County Hospital. The mean age was 40 years and 68 patients (64\%) were male. The mean TBSA was $30 \%$ for all burn victims. Of these patients, $14 \%$ had TBSA $\geq 50 \%$. 32 patients $(30 \%)$ had concomitant inhalation injuries. The mean Baux score and R-Baux was 70 and 75, respectively. The mean pulse pressure and shock index on admission for all patients were $59 \mathrm{mmHg}$ and 0.81 respectively. The overall mortality rate was $17 \%$ (18/108), with a total of 90 survivors. 
The mean age of the survivors compared to non-survivors was 38 years and 51 years, respectively $(p=$ 0.0294). The mean TBSA in survivors versus non-survivors was $24.2 \%$ and $58.5 \%$, respectively $(p<0.001)$. Among eighteen non-survivors, fourteen $(78 \%)$ had inhalation injury $(p<0.001)$. Non-survivors had higher mean Baux score and R-Baux compared to survivors (Baux 109.6 vs 62.2, and R-Baux (122.8 vs 64.6, $p<$ $0.001)$. There was no significant difference in the mean heart rate between survivors (98bpm) and nonsurvivors $(113 \mathrm{bpm}),(p=0.0376)$ on admission. There was also no significant differences between survivor and non-survivor mean SBP $(134 \mathrm{mmHg}$ vs. $121 \mathrm{mmHg}, \mathrm{p}=0.1023)$ and DBP $(73 \mathrm{mmHg}$ vs. 74 $\mathrm{mmHg} \mathrm{p}=0.869$ ), respectively.

Survivors had a higher mean pulse pressure as compared to non-survivors $(61 \mathrm{mmHg}$ vs $47 \mathrm{mmHg}, \mathrm{p}=$ $0.0051)$. A higher mean shock index was associated with non-survivors vs survivors ( 1.0 vs $0.77, p=$ $0.006)$. Non-survivors were more likely to have elevated shock index values $(\mathrm{SI} \geq 0.9)$ as compared to normal shock index values $(<0.9)$ in the survivor group $(p<0.0001)$.

Multivariate logistic regression analysis showed that the adjusted odds ratio for R-Baux and shock index were 1.06 (1.03-1.09) and 26.48 (4.34-161.46). For every unit increase in shock index, the odds of mortality increased by 26 -fold.

\section{Discussion}

Although burns are common injuries, the overall mortality rate is relatively low ${ }^{2}$. In fact the ABA quoted that the survival rate for burn injury patients in the U.S. is $96.8 \%{ }^{2}$. Similar to our study's relatively high survival rate of $83 \%$, we hypothesized that vital signs on admission would help predict and identify patients with a low likelihood of survival.

Although our sample of 108 patients was small, the results of our study can be generalized for patients with significant burn injuries. Our data analysis demonstrated initial vital signs such as heart rate, systolic and diastolic blood pressures were not significantly different between survivors and nonsurvivors with a TBSA $\geq 15 \%$ and had no predictive value for mortality. However, shock index and pulse pressure were significant predictors of mortality for these patients.

Shock index is fast, reliable and useful prognostic tool for predicting mortality in acute trauma, hemorrhagic or septic patients ${ }^{18}$, however the use of shock index in burn patients' mortality risk remains unknown. This is the first study to explore the relationship between shock index and clinical outcome in burns victims. SI has been suggested to be a reliable and reproducible screening tool for identifying patients at risk for shock despite apparent hemodynamic stability ${ }^{19}$. Blood pressure and pulse are often normal during the initial compensatory phase of shock and can therefore underestimate the severity of hemodynamic instability ${ }^{20}$. An abnormal shock index can serve as early identifier of patients that require prioritization for medical care. In fact, studies have demonstrated that SI as compared to traditional vital signs is superior at early identification and predicting mortality outcomes in at risk patients ${ }^{15}$. Evidence has shown that normal SI range is $0.5-0.7$, with 0.9 accepted as the upper limit of normal ${ }^{20}$. In all 
trauma patients excluding those with traumatic brain injury, a $\mathrm{SI} \geq 0.9$ predicts mortality ${ }^{19}$; however, other studies have also shown that $\mathrm{SI}>1.0$ predict an increased risk of mortality and hemodynamic collapse ${ }^{20}$. Similarly, significant burn injuries compromise hemodynamic stability resulting in occult shock ${ }^{11}$. Our results corroborated this theory, as the mean $\mathrm{SI}$ in non-survivors and survivors was 1.0 and 0.7 , respectively. For this reason, an elevated $\mathrm{SI}$ in burn patients can be used to identify victims at risk for hemodynamic compromise and a higher likelihood of mortality so that early treatment and resuscitation efforts can be directed at those with poor predicted clinical outcome, and to improve survival rates.

In addition, adjusted odds ratio as shown in Table 2 for R-Baux score and shock index were 1.06 and 26.48 , respectively. Our results demonstrated that for every unit increase in shock index, the odds of mortality increased by 26 -fold. These results are significant because one of the most frequently utilized mortality prediction models in burn patients is the R-Baux score ${ }^{21}$. The R-Baux score originates from the Baux score, a prognostic tool relying solely on TBSA and age to estimate mortality outcomes in burn victims ${ }^{21}$. However, the revised score which includes the inhalation injury status has been shown to be a more accurate predictor ${ }^{21}$ due to higher likelihood of hemodynamic compromise associated with the presence of inhalation injury ${ }^{22}$. As suggested by the R-Baux score, mortality is a more likely outcome in patients with advanced age, larger burn size and presence of inhalational injury ${ }^{21}$. As shown in Table 1, this was consistent with our findings; however, mortality outcomes in burn patients is dependent on the degree of hemodynamic instability ${ }^{11}$ which initial physiologic parameters such as shock index may have better discriminatory ability ${ }^{23}$ as compared to the R-Baux score. 
Table 1

Comparison of variables between the mortality status in burn patients

\begin{tabular}{|c|c|c|c|c|}
\hline Variables & Overall $(n=108)$ & Dead $(n=18)$ & Alive $(n=90)$ & p-value \\
\hline Age (years) & $40.23 \pm 23.2$ & $51.06 \pm 23.98$ & $38.07 \pm 22.56$ & 0.0294 \\
\hline Gender & & & & 0.7584 \\
\hline Female & 36 & $5(31.3 \%)$ & $31(35.2 \%)$ & \\
\hline Male & 68 & $11(68.8 \%)$ & $57(64.8 \%)$ & \\
\hline TBSA & $29.93 \pm 21.17$ & $58.56 \pm 28.26$ & $24.2 \pm 13.69$ & $<.0001$ \\
\hline TBSA category & & & & $<.0001$ \\
\hline TBSA $<50 \%$ & 93 & $7(38.9 \%)$ & $86(95.6 \%)$ & \\
\hline $\mathrm{TBSA} \geq 50 \%$ & 15 & $11(61.1 \%)$ & $4(4.4 \%)$ & \\
\hline Inhalation Injury & & & & $<.0001$ \\
\hline No & 74 & $4(22.2 \%)$ & $70(79.6 \%)$ & \\
\hline Yes & 32 & $14(77.8 \%)$ & $18(20.5 \%)$ & \\
\hline Shock Index & $0.81 \pm 0.33$ & $1 \pm 0.39$ & $0.77 \pm 0.3$ & 0.006 \\
\hline Shock Index Category & & & & $<.0001$ \\
\hline Shock Index $<0.9$ & 75 & $5(27.8 \%)$ & $70(77.8 \%)$ & \\
\hline Shock Index $\geq 0.9$ & 33 & $13(72.2 \%)$ & $20(22.2 \%)$ & \\
\hline Baux score & $70.16 \pm 34.2$ & $109.61 \pm 35.47$ & $62.27 \pm 28.07$ & $<.0001$ \\
\hline R score & $0.32 \pm 0.47$ & $0.78 \pm 0.43$ & $0.22 \pm 0.42$ & $<.0001$ \\
\hline Revised Baux score & $75.12 \pm 38.74$ & $122.83 \pm 37.05$ & $64.64 \pm 30.48$ & $<.0001$ \\
\hline $\mathrm{SBP}(\mathrm{mmHg})$ & $131.52 \pm 30.84$ & $120.67 \pm 36.73$ & $133.69 \pm 29.28$ & 0.1023 \\
\hline $\mathrm{DBP}(\mathrm{mmHg})$ & $72.94 \pm 20.2$ & $73.67 \pm 22.78$ & $72.8 \pm 19.79$ & 0.869 \\
\hline HR (bpm) & $100.68 \pm 28.01$ & $113.17 \pm 29.68$ & $98.18 \pm 27.15$ & 0.0376 \\
\hline Pulse Pressure & $58.57 \pm 19.43$ & $47 \pm 20.52$ & $60.89 \pm 18.46$ & 0.0051 \\
\hline
\end{tabular}


Table 2

Odds ratio and $95 \%$ confidence interval for predicting mortality

\begin{tabular}{|lll|}
\hline Predictors & Unadjusted OR & Adjusted OR \\
\hline Age & $1.03(1,1.05)$ & \\
\hline TBSA $\geq 50 \%$ vs TBSA $<50 \%$ & $33.79(8.5,134.23)$ & \\
\hline Pulse pressure & $0.96(0.93,0.99)$ & \\
\hline Shock Index $\geq 0.9$ vs Shock Index $<0.9$ & $9.1(2.9,28.59)$ & $26.48(4.34,161.46)$ \\
\hline Inhalation Injury: Yes vs No & $13.61(3.99,46.38)$ & \\
\hline Revised Baux score & $1.05(1.03,1.07)$ & $1.06(1.03,1.09)$ \\
\hline
\end{tabular}

Pulse pressure is another early indicator of hemodynamic instability, and it begins to narrow in the early stages of compensated shock in seemingly asymptomatic patient. Priestley et al demonstrated that relatively narrowed pulse pressures are independent early predictors of hemorrhage in acute trauma patients ${ }^{24}$. Similar to hemorrhage induced hypovolemic shock, severe burn injury is associated with early hypovolemic shock resulting in low cardiac output with corresponding reduction of both preload and stroke volume that in return decreases the pulse pressure ${ }^{25}$. Our results showed significant difference in mean pulse pressure between survivors $(61 \mathrm{mmHg})$ and non-survivors $(47 \mathrm{mmHg})$. Although normal range of pulse pressure is $40-60 \mathrm{mmHg}$ in healthy adults; our study suggests that a relatively decreased pulse pressure in burn victims can be used to identify victims associated with a higher probability of mortality in the early stages of shock to improve survival outcomes.

Shock index and pulse pressure are useful and quick tools that can be utilized by health-care professionals to identify seemingly stable patients who may benefit from early and aggressive fluid resuscitation to prevent occult shock and death. In addition, these findings may help guide clinical decisions and medical management for acute burn injury patients in the hospital setting.

\section{Conclusions}

Although patients with severe burns may present with similar hemodynamic vital signs on admission, pulse pressure and shock index have prognostic value and can assist clinical decision making in the acute burn setting. Higher shock index and relatively lower pulse pressures on admission are early predictors of in-hospital mortality. Shock index is associated with 26 times higher likelihood of mortality and should be utilized as a prognostic tool for predicting mortality outcomes in burn patients.

\section{Abbreviations}

\section{ABA}

American Burn Association

DBP 
Diastolic Blood Pressure

HR

Heart Rate

IL-6

Interleukin-6

P-Baux

Pediatric-Baux score

PP

Pulse Pressure

R-Baux

Revised Baux score

SI

Shock Index

SBP

Systolic Blood Pressure

TBSA

Total Body Surface Area

TNF-a

Tumor Necrosis Factor alpha

\section{Declarations}

Ethics Approval and Consent to Participate: This study obtained approval from Cook County Hospital IRB \# 19-154. Human consent was waived due to the nature of the retrospective study and de-identification of the data.

Consent for Publication: Not applicable because there are no individually detailed data, videos or images.

Availability of Data and Materials: The dataset supporting the conclusion of this manuscript is included as an additional file uploaded as a supporting document for reviewers.

Competing Interests: The authors declare that they have no competing interests.

Funding: Not applicable

Author's Contributions: FB, SP and APM were equally involved with acquisition of data and initial conception of the study. RJ substantively revised the design of the project. FD and APM performed statistical analysis with STATA software and R-commander, respectively in addition to providing the tables and figures included in the manuscript. DL was equally involved with data interpretation and manuscript production. APM was a major contributor in writing the manuscript. All authors read and approved the final manuscript. 
Acknowledgements: Not Applicable

\section{References}

1. Burns." World Health Organization, World Health Organization, 6 Mar. 2018, www.who.int/newsroom/fact-sheets/detail/burns.

2. "Burn Incidence Fact Sheet." American Burn Association, American Burn Association, 8 May 2017, ameriburn.org/who-we-are/media/burn-incidence-fact-sheet/.

3. "Burn Injury Fact Sheet." National Burn Awareness Week, American Burn Association, 4 Feb. 2018, ameriburn.org/wp-content/uploads/2017/12/nbaw-factsheet_121417-1.pdf.

4. Schaefer, Timothy J., and Shruti C. Tannan. "Thermal Burns." StatPearls [Internet]., U.S. National Library of Medicine, 14 Feb. 2019, www.ncbi.nlm.nih.gov/books/NBK430773/.

5. "Burns." CDC Injury Prevention, CDC. https://www.cdc.gov/masstrauma/factsheets/public/burns.pdf

6. Hettiaratchy, Shehan, and Peter Dziewulski. "ABC of Burns: Pathophysiology and Types of Burns." BMJ (Clinical Research Ed.), BMJ Publishing Group Ltd., 12 June 2004, www.ncbi.nlm.nih.gov/pmc/articles/PMC421790/.

7. Nielson, Colton B, et al. "Burns: Pathophysiology of Systemic Complications and Current Management." Journal of Burn Care \& Research : Official Publication of the American Burn Association, Lippincott Williams \& Wilkins, Jan. 2017, www.ncbi.nlm.nih.gov/pmc/articles/PMC5214064/.

8. Abu-Sittah, G S, et al. "Cardiovascular Dysfunction in Burns: Review of the Literature." Annals of Burns and Fire Disasters, 2012 Mar 31, 25(1): 26-37 PubMed PMID: 23012613.

9. Jeschke, Marc G, et al. "Burn Injury." Nature Reviews. Disease Primers, Nature Publishing Group UK, 13 Feb. 2020, www.ncbi.nlm.nih.gov/pmc/articles/PMC7224101/.

10. Schaefer, Timothy J., and Omar Nunez Lopez. "Burn Resuscitation And Management." StatPearls [Internet]., U.S. National Library of Medicine, 10 Mar. 2019, www.ncbi.nlm.nih.gov/books/NBK430795/.

11. Soussi, Sabri, et al. "Early Hemodynamic Management of Critically III Burn Patients." Anesthesiology, vol. 129, no. 3, 2018, pp. 583-589., doi:10.1097/aln.0000000000002314.

12. Soussi, Sabri, et al. "Low Cardiac Index and Stroke Volume on Admission Are Associated with Poor Outcome in Critically III Burn Patients: a Retrospective Cohort Study." Annals of Intensive Care, 2016 Dec, 6(1), 87 PubMed DOI: 10.1186s13613-016-0192-y.

13. Haberal, Mehmet, et al. "Fluid Management in Major Burn Injuries." Indian Journal of Plastic Surgery, 2010 Sept, 43(Suppl): S29-S36 PubMed DOI: 10.4103/0970-0358.70715.

14. Berger, Tony, et al. "Shock Index and Early Recognition of Sepsis in the Emergency Department: Pilot Study." The Western Journal of Emergency Medicine, Department of Emergency Medicine, University of California, Irvine School of Medicine, Mar. 2013, https://www.ncbi.nlm.nih.gov/pmc/articles/PMC3628475/. 
15. Kamikawa, Yohei, and Hiroyuki Hayashi. "Equivalency between the Shock Index and Subtracting the Systolic Blood Pressure from the Heart Rate: An Observational Cohort Study." BMC Emergency Medicine, BioMed Central, 31 Oct. 2020, https://www.ncbi.nlm.nih.gov/pmc/articles/PMC7603662/.

16. Mistovich, Joseph. "Blood Pressure Assessment in the Hypovolemic Shock Patient." EMS1, 18 Jan. 2018, www.ems1.com/ems-products/ambulance-disposable-supplies/articles/blood-pressureassessment-in-the-hypovolemic-shock-patient-X0297tdQwsnwrVD7/.

17. Tsurumi, A., Que, Y., Yan, S., Tompkins, R., Rahme, L. and Ryan, C., 2015. Do standard burn mortality formulae work on a population of severely burned children and adults?. Burns, 41(5), pp.935-945.

18. Montoya, Kevin, et al. "Shock Index as a Mortality Predictor in Patients with Acute Polytrauma." Journal of Acute Disease, 2015 July, 4(3): 202-204 Elsevier DOI: 10.1019/j.joad.2015.04.006.

19. Odom, Stephen R, et al. "Extremes of Shock Index Predicts Death in Trauma Patients." Journal of Emergencies, Trauma, and Shock, 2016 Jul-Sept, 9(3): 103-106 PubMed DOI: 10.4103/09742700.185272.

20. Koch, Erica, et al. "Shock Index in the Emergency Department: Utility and Limitations." Open Access Emergency Medicine, Volume 11, 2019, pp. 179-199., doi:10.2147/oaem.s178358.

21. Williams, D.j., and J.d. Walker. "A Nomogram for Calculation of the Revised Baux Score." Burns, vol. 41, no. 1, 2015, pp. 85-90., doi:10.1016/j.burns.2014.05.001.

22. Woodson, L., Talon, M., Traber, D. and Herndon, D., 2021. Diagnosis and treatment of inhalation injury. [online] Plastic Surgery Key. Available at: <https://plasticsurgerykey.com/diagnosis-andtreatment-of-inhalation-injury/> [Accessed 20 May 2021].

23. El Ayadi, A., Nathan, H., Seed, P., Butrick, E., Hezelgrave, N., Shennan, A. and Miller, S., 2016. Vital Sign Prediction of Adverse Maternal Outcomes in Women with Hypovolemic Shock: The Role of Shock Index. PLOS ONE, [online] 11(2), p.e0148729. Available at: $<$ https://pubmed.ncbi.nlm.nih.gov/26901161/>.

24. Priestley, Erika M., et al. "Pulse Pressure as an Early Warning of Hemorrhage in Trauma Patients." Journal of the American College of Surgeons, vol. 229, no. 2, 2019, pp. 184-191., doi:10.1016/j.jamcollsurg.2019.03.021.

25. Homan, Travis D., and Erica Cichowski. "Physiology, Pulse Pressure." StatPearls [Internet]., U.S. National Library of Medicine, 25 Apr. 2019, www.ncbi.nlm.nih.gov/books/NBK482408/. 